\title{
Short-Term Longitudinal Study of Central Auditory Function in Alzheimer's Disease and Mild Cognitive Impairment
}

\author{
Esma Idrizbegovic Christina Hederstierna Martin Dahlquist \\ Ulf Rosenhall \\ Department of Audiology and Neurotology, Karolinska University Hospital, \\ Stockholm, Sweden
}

\author{
Key Words \\ Central auditory processing $\cdot$ Dementia $\cdot$ Hearing
}

\begin{abstract}
Background/Aims: Central auditory function can be studied to monitor the progression of mild cognitive impairment to dementia. Our aim was to address this issue in a prospective longitudinal setting. Methods: Tests of central hearing function were performed on 70 subjects with either Alzheimer's disease (AD) or mild cognitive impairment, and in controls with subjective memory complaints but normal cognition. The time span until follow-up was 1.5 years. Results: The dichotic digit free recall test showed a significant decline in the AD group compared with the controls (left ear). Conclusion: The short time span was long enough to disclose a central auditory processing decline in AD.

(c) 2013 S. Karger AG, Basel
\end{abstract}

\section{Introduction}

Central auditory processing (CAP) dysfunction has been described in Alzheimer's disease (AD), and has been demonstrated even in mild cognitive impairment (MCI) [1-3]. Gates et al. [3] showed that tests of the central auditory function can be used to predict the development of incipient dementia. In their study, a cohort of elderly persons was tested at one occasion. Those who later developed AD had significantly poorer initial CAP function than the others. Furthermore, the CAP function deteriorates with age [1].

Within the framework of an investigation of central and peripheral hearing in AD and $\mathrm{MCI}$, a baseline study has been performed [2]. A cohort of persons with definite or suspected cognitive or memory problems was included. The AD patients were at an early stage. MCI patients had memory problems and cognitive decline, but they did not fulfill the requirements 
Table 1. Number of invited subjects, and number of participants in the longitudinal study at follow-up

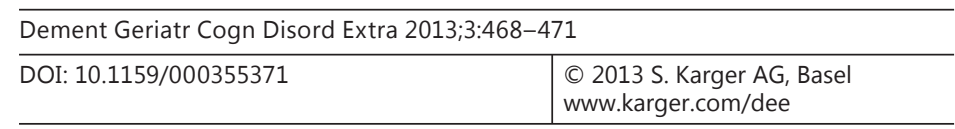

Idrizbegovic et al.: Short-Term Longitudinal Study of Central Auditory Function in Alzheimer's Disease and Mild Cognitive Impairment

\begin{tabular}{lrrrr}
\hline & All & AD & MCI & SMC \\
\hline Subjects at baseline, n & 104 & 32 & \multicolumn{1}{c}{40} & \multicolumn{1}{c}{32} \\
Subjects at follow-up, n & 70 & 17 & 25 & 28 \\
Response at follow-up, \% & 67.3 & 53.1 & 62.5 & 87.5 \\
Age at follow-up, years & & 64.9 & 61.7 & 63.5 \\
Follow-up period, years & & 1.3 & 1.5 & 1.5 \\
\hline
\end{tabular}

for the diagnosis of dementia, and they had intact activities of daily living. The controls had subjective memory complaints but normal cognition (SMC group). Our results demonstrated that CAP dysfunction was evident especially in $\mathrm{AD}$, but also in $\mathrm{MCI}$ [2]. Two follow-ups were scheduled, the first 1.5 years after the baseline study, and the second 5 years after baseline. To our knowledge, there are no other systematic longitudinal studies on CAP function in these conditions.

\section{Subjects and Methods}

The participants of the initial study $(n=104)$ were invited to the first follow-up approximately 1.5 years after the baseline investigation. At the follow-up, 70 participants $(67.3 \%$; 40 women) completed the test protocol (table 1). Data regarding the number of subjects and age are presented in table 1. The follow-up was performed from April 2008 to September 2010. The design of the study was prospective and longitudinal. The study was approved by the regional ethics review board.

\section{Cognitive Development of the Three Subgroups}

Four of the participants developed dementia during the study period, i.e. three from the MCI group and one from the SMC group. Three of these subjects had developed AD, and one of them Pick's disease. Eight with MCI had improved and were regarded as SMC subjects at the follow-up. One subject with SMC had developed MCI. The other 57 subjects did not change the subgroup affiliation from baseline to follow-up.

\section{Pure Tone and Speech Audiometry}

Pure tone audiometry including air conduction thresholds at $0.125-8 \mathrm{kHz}$ and bone conduction at $0.25-4 \mathrm{kHz}$ was performed, according to ISO 8253-3 [4], using a GN Resound Orbiter 922 version 2 audiometer and TDH-39 ear phones in a sound-attenuated booth. The speech-in-noise (SPIN) test $(\mathrm{S} / \mathrm{N}+4 \mathrm{~dB})$ consisted of phonemically balanced monosyllabic words in Swedish, according to Magnusson [5]. The Wilcoxon matched-pairs test was used for statistical analysis. Predicted values according to age and high-frequency hearing were calculated in keeping with a model described by Barrenäs and Wikström [6].

Dichotic digits tests (DDT) were presented in lists containing series of two digits, according to a previously described Swedish test protocol [7]. The 2-digit tests were performed under two different conditions: (1) directed report, where the subject was asked to repeat what was heard only in the right or only in the left ear, respectively, and (2) free report, where the subject was asked to repeat what was heard in both ears, without specifying in which ear it was heard. Only the DDT 2-digit test results were considered, since the 3-digit test results were judged to be noninformative in the baseline study [2]. Kruskal-Wallis ANOVA with multiple comparisons of mean ranks was used for between-group analyses. All tests were performed by a trained clinical audiologist. 
Fig. 1. DDT scores (percent correct responses), free report (FR) situation, left ear (LE) at baseline and at follow-up are shown. The three subgroups (AD, MCI, SMC) include those who did not change subgroup affiliation during the study.

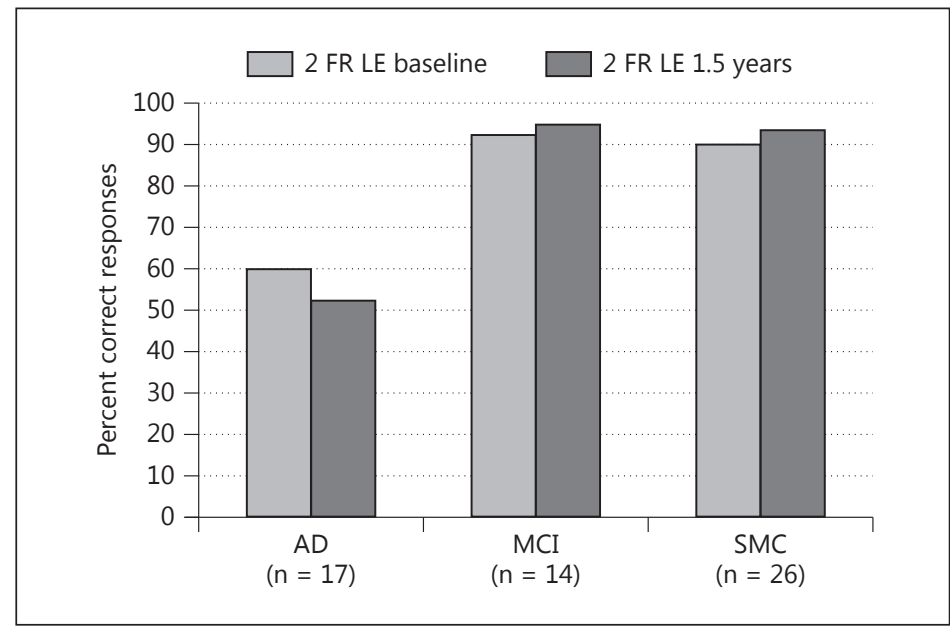

\section{Results}

\section{Pure Tone and Speech Audiometry}

At follow-up, the median pure tone thresholds showed no significant decrease in the AD and the MCI groups; in the SMC group, there were significant, although small (2.5-5 dB and $10 \mathrm{~dB}$ at only one frequency) decreases in several of the high frequencies in both ears. The decline is in accordance with what is expected for the age of the subjects, and there were no significant between-group differences. The SPIN test showed a significant decline in both ears in the AD group, but only in the left ear in the other groups. The median SPIN values were all still within the predicted limits, adjusting for age and high-frequency hearing.

\section{Dichotic Digits Tests}

Free Report, Left Ear. The AD group demonstrated a significant median score decline $(\mathrm{p}=0.03)$, and there was a significant median score improvement $(\mathrm{p}=0.01)$ in the SMC group (fig. 1). This short-term longitudinal change of the DDT scores, over 1.5 years, differed significantly between the AD and the SMC groups $(\mathrm{p}=0.006)$, but not between the MCI group and the two other groups.

Free Report, Right Ear and Directed Report, Both Ears. No significant changes were seen between the baseline and follow-up, neither within nor between any of the three groups.

\section{Discussion}

Humes et al. [8] concluded that central presbycusis exists as a multifactorial condition related to age and/or disease changes in the auditory system and in the brain. Tests of central auditory function can be used to predict which individuals might be at risk of developing dementia [1,3]. The DDT is a test of central auditory function, and the AD group deviated significantly from the control group of similar age, in accordance with a pattern that had been described earlier [2]. On a group level, the nondominant left ear performed significantly worse in both the free report and the directed report situations at both test sessions. After 1.5 years, there was a significant decline of the DDT performance (free report, left ear) in the AD group. There was no significant decline regarding the right ear, or in the directed report situation in either ear. It is conceivable that the decline is more pronounced in the left ear than 
in the right, and also in the more difficult free report situation. These findings, as well as that of equally affected SPIN scores in all three groups, indicate that the instructions were understood, and that the results imply a real CAP decline in the AD group. The decline of the pure tone thresholds and the SPIN test during the follow-up period is consistent with what can be expected in the general population of the corresponding age.

The MCI and SMC groups had no apparent DDT decline during the study period. On the contrary, there was a slight tendency in the opposite direction, which can be explained by a training effect. Gates et al. [3] reported poorer CAP performance in a group who later developed dementia. Their study had a cross-sectional design, which permits the inclusion of a large study group. The longitudinal design of our study group meant that many patients belonged to a different group at the 1.5-year follow-up, and we only calculated if DDT scores had changed significantly in those that remained in the same groups. However, the four patients that developed dementia during the study period showed DDT results similar to those of the AD group.

We could not observe any CAP decline in the MCI group during the short follow-up period. This issue will be investigated further in the planned, 5-year follow-up study.

The rationale for using a short follow-up period, i.e. 1.5 years, was the assumption that $\mathrm{AD}$ patients could be difficult to mobilize for participation in a study with a longer follow-up interval. This presumption turned out to be correct. The short time span was long enough to disclose a CAP decline in the AD group. Our conclusion is that the DDT is easy and time-efficient to perform and is a suitable test for the purpose of monitoring the progress of AD.

\section{Acknowledgements}

The work was supported by the Regional Agreement on Medical Training and Clinical Research (ALF) between the Stockholm County Council and the Karolinska Institutet, and by grants from the Foundation 'Tysta Skolan'. Neither of these sponsors played any role in design, execution, analysis interpretation or writing of the article. We would also like to thank Ann Ålander, clinical audiologist, for her professional work with testing all subjects.

\section{References}

1 Gates GA, Anderson ML, Feeney MP, McCurry SM, Larson EB: Central auditory dysfunction in older persons with memory impairment or Alzheimer dementia. Arch Otolaryngol Head Neck Surg 2008;134:771-777.

-2 Idrizbegovic E, Hederstierna C, Dahlquist M, Kampfe Nordstrom C, Jelic V, Rosenhall U: Central auditory function in early Alzheimer's disease and in mild cognitive impairment. Age Ageing 2011;40:249-254.

-3 Gates GA, Anderson ML, McCurry SM, Feeney MP, Larson EB: Central auditory dysfunction as a harbinger of Alzheimer dementia. Arch Otolaryngol Head Neck Surg 2011;137:390-395.

4 International Organization for Standardization: ISO 8253-3:1996. Acoustics - Audiometric Test Methods Part 3: Speech Audiometry. Geneva, International Organization for Standardization, 1996.

5 Magnusson L: Reliable clinical determination of speech recognition scores using Swedish PB words in speechweighted noise. Scand Audiol 1995;24:217-223.

6 Barrenäs ML, Wikström I: The influence of hearing and age on speech recognition scores in noise in audiological patients and in the general population. Ear Hear 2000;21:569-577.

7 Hallgren M, Larsby B, Lyxell B, Arlinger S: Cognitive effects in dichotic speech testing in elderly persons. Ear Hear 2001;22:120-129.

-8 Humes LE, Dubno JR, Gordon-Salant S, Lister JJ, Cacace AT, Cruickshanks KJ, Gates GA, Wilson RH, Wingfield A: Central presbycusis: a review and evaluation of the evidence. J Am Acad Audiol 2012;23:635-666. 